\title{
Characteristics of supination-external rotation Salter-Harris II ankle fractures in computed tomography
}

Yunfang Zhen ( $\square$ yfzhen@suda.edu.cn )

\section{Research article}

Keywords: Distal tibial physeal fracture, Ankle fractures, Salter-Harris type II fracture, fibular fracture, Computed tomography, Children

Posted Date: October 14th, 2021

DOI: https://doi.org/10.21203/rs.3.rs-968616/v1

License: (c) (1) This work is licensed under a Creative Commons Attribution 4.0 International License. Read Full License 


\section{Abstract}

Background: Recent literature has shown that Salter-Harris (S-H) $ه$ fractures are the most common ankle fractures and carry a higher rate of growth disturbance. Recent literature has shown that Salter-Harris (S$\mathrm{H}) \otimes$ fractures are the most common ankle fractures. CT characteristics of S-H $\otimes$ ankle fractures are not well depicted. The purpose of this study was to evaluate supination-external rotation (SER) S-H囚ankle fractures by CT and to analyze the features of the associated fibular fracture to further determine the injury mechanism.

Methods: The radiographs and CT with S-H \ankle fractures were reviewed. Patients suffered from SER injury were included. The medial tibial cortex (MTC) of the distal tibia broken or intact, the metaphyseal fracture angle (MFA) $5-10 \mathrm{~mm}$ proximal to the physis was documented in axial CT. The length of the metaphyseal fragment was measured in saggital CT. The correlation of the upper limits between fibular fracture and metaphyseal fragment was analyzed. In presence of the fibular fracture, the fracture pattern was classified based on the location and morphology of the fracture line.

Results: Seventy-nine SER S-H囚ankle fractures were identified. Stage 1 was present in 35 and stage 2 in 44. In axial CT, the mean MFA was 11.2 degrees. MTC was fully broken in one case and 20, in stage 1 and stage 2, respectively $(P=0.001)$. In saggital $C T$, the mean length of metaphyseal fragment was $35.3 \mathrm{~mm}$. The length of this fragment was $35.0 \mathrm{~mm}, 35.5 \mathrm{~mm}$, in stage 1 and stage 2 , respectively $(P=0.868)$. The upper part of the fibular fracture line was located at the same level or higher than that of metaphyseal fragment. In 44 cases with associated fibular fracture, forty were in distal metaphysis with oblique fracture line for which 4 types were demonstrated with plantar flexion. Other 4 were in distal diaphysis with spiral fracture line.

Conclusions: For SER S-H $\otimes$ ankle fractures, MTC and orientation of the fracture plane can be shown in CT to help to make an appropriate preoperative plan. In addition to SER, majority of the concurrent fibular fracture was in the distal metaphysis with oblique fracture line and plantar flexion.

\section{Background}

Pediatric ankle fractures constitute $15-20 \%$ of all physeal injuries [1]. Type $\otimes$ fractures are the most common ankle physeal fractures, based on Salter-Harris (S-H) classification [2], which accounted for 32$60 \%$ of the distal tibial physeal fractures [3,4]. The supination-external rotation (SER) injury, according to the Dias-Tachdjian classification [5], was the most common deforming mechanism to result in $\mathrm{S}-\mathrm{H} \mathbb{\nabla}$ ankle fractures in children [6]. Moreover, Spiegel et al. [7] believed that the risk factor of premature physeal closure for $\mathrm{S}-\mathrm{H} \otimes$ ankle fractures was unpredictable.

Traditionally, conventional radiographs (CR) can demonstrate most of information in terms of classification purpose and is considered as the initial diagnostic stool for ankle fractures $[8,9]$. However, the precise fracture pattern or exact localization of the fragment could not be determined on CR in all cases [10]. Computed tomography (CT) scans have been proven to reveal the accurate fracture pattern 
for complicated ankle fractures, to provide necessary information not available on CR and to make an optimal decision of treatment $[9,11,12]$. Moreover, in a cadaver study on Tillaux fracture by Horn et al, CT scans were more sensitive than $\mathrm{CR}$ at identifying fractures with displacement of $>2 \mathrm{~mm}$ [13]. Both the screw position and trajectory determined by CR only may be altered after reviewing $\mathrm{CT}$ in case of reduction and internal fixation[12,14].

Previous studies have focused on the treatment of choice and the complications for this type fracture $[3,4,15,16]$. Generally, fracture lines in posterior metaphysis may be a challenge to be identified by preoperative CR or intraoperative fluoroscopy. However, CT has not been used systematically to address the features with respect to $\mathrm{S}-\mathrm{H} \otimes$ ankle fractures. In present study, we analyzed $\mathrm{S}-\mathrm{H} \otimes$ ankle fractures treated in our institution and depicted the characteristics demonstrated on CT to improve the ability of understanding the given fractures.

\section{Methods}

\section{Patients}

After institutional review board approval, we retrospectively reviewed radiographic and clinical records of the children presenting with ankle fractures from January 2015 to December 2020. In our institution, conventional anteroposterior (AP) and lateral X-rays were made in every patient as well as CT scan of the injured ankle in case of the displacement of $3 \mathrm{~mm}$ or greater preoperatively. Patients were included if they had a partially or completely open growth plate and underwent CT scan of the injured ankle. Patients in whom the growth plate was completely closed, CT was unavailable or with concurrent ipsilateral tibial shaft fracture were excluded.

The SER mechanism of injury was defined based on the Dias-Tachdjian system [5] and two pediatric orthopaedists and one radiologist retrospectively detected all the CR and CT images independently and blinded to measure the parameters. The mean value from the three observers were calculated. When the observers disagreed, one senior pediatric orthopedist and radiologist reviewed the images and performed the final interpretation.

CT technique

All ankle CT examinations were performed on 32 or 64-row scanner. On average, the technique for acquiring helical images used 60-100 kVp and 60-80 mAs. Every slice had widths between $2 \mathrm{~mm}$ and 5 $\mathrm{mm}$. All the radiographic images were retrieved and measurements were subsequently carried out using PACS software.

Radiographic data were recorded as following:

1) medial tibial cortex (MTC) intact or not (Fig 1). The distal tibial metaphysis which was not penetrated completely in axial CT scans or reconstruction views was considered as intact MTC. 
2) metaphyseal fracture angle (MFA) (Fig.1-C), which comprised two lines, alone the anterior tibial edge and alone the proximal metaphyseal fracture plane. In the axial plane through the metaphysis, fracture line was identified $5-10 \mathrm{~mm}$ proximal to the physis, in that it is the typical level in case of internal fixation of screws to stabilize the fracture[16,17] (Fig.2). The anterior tibial line was drawn along the anterior edge of the tibia and the fracture line was drawn along the posterior edge of the distal tibial metaphysis. The angle between the two lines aforthmentioned was used to guide the optimal direction of the screws.

3) length of Thurston-Holland (TH) metaphyseal fragment, which was defined as the vertical height between the distal tibial growth plate and the highest point of the fragment.

4) associated fibular fracture. In the presence of the associated fibular fracture, the fracture pattern was classified based on the location and the morphology of the fracture in reconstructive CT views. The correlation of the upper limits between the fibular fracture and the TH fragment was classified as: at the same level of both limits, at higher or lower level of the fibular fracture than that of $\mathrm{TH}$ fragment.

Statistical Analysis

Data were analyzed using SPSS Statistics for Windows software, version 20.0 (SPSS Inc., Chicago, IL). Two-tailed $P$-value less than 0.05 was considered statistically significant. Continuous variables are displayed as the mean $\pm S D$ and categoric variables as counts and percentages. Pearson chi-square analysis was used for categorical variables and student $t$ tests were conducted to compare continuous variables.

\section{Results}

Patient demographic information is summarized in Table 1. A total of 79 SER S-H $\otimes$ ankle fractures with available CT scans were identified in 79 children, 27 girls and 52 boys. The right ankle was injured in 45 cases (57\%), and the left in $34(43 \%)$. The mean age of these patients was $12.2 \pm 1.4$ years (range, 8-14.6), $11.6 \pm 1.2$ years for girls and $12.5 \pm 1.4$ years for boys. The age difference in our series was statistically significant $(P=0.008)$. According to the Dias-Tachdjian staging for SER injury, in 35 of our 79 cases stage 1 was present and stage 2 was present in the remaining 44 cases.

In the axial plane, the lateral cortical bone was fully fractured and the $\mathrm{TH}$ fragment was rotational posterolaterally in all of the 79 cases. The mean MFA was 11.2 \pm 9.1 degrees (ranged, $1-43$ degrees), $11.4 \pm 8.5$ degrees in stage 1 and $11.1 \pm 9.6$ degrees in stage 2 . There was no significant difference $(P=0.861)$. This angle was 20 degrees or greater in 11 cases. The condition of MTC, along with the stage of injury, are as follows: stage 1, intact in 34 cases, completely fractured in 1; stage 2, intact in 24 cases, completely fractured in 20 . There was significant difference $(P=0.001)$ for fully fractured medial cortex between stage 1 and stage 2 .

In the saggital plane, the mean size of the TH fragment was $35.3 \pm 12.1 \mathrm{~mm}$ (ranged 15 to $65 \mathrm{~mm}$ ), $35.0 \pm 11.2 \mathrm{~mm}$ in stage 1 and $35.5 \pm 12.8 \mathrm{~mm}$ in stage 2 . There was no significant difference $(P=0.868)$. 
The upper part of the fibular fracture line was located at the same level as that of the TH fragment in 27 patients and higher than that in 17 (Fig. 3). Table 2 summarized the difference of the several parameters between stage 1 and stage 2 .

Fibular fractures were associated in 44 cases. In 14 cases, fibular fracture was not initially shown on CR, but was detected in CT scans (Fig 4). Forty out of the associated fibular fractures were in the distal metaphysis and other 4 in the distal diaphysis. For those that involved the metaphysis, the fracture line was of long obliquity, extending from the anterior edge in a posterosuperior direction. The distal end of the fracture was situated at the level or proximal to the fibular epiphysis, all being at the same level as the tibial physis. Plantar flexion of the anterior cortex was observed with an apex dorsally and, when present, with a typical splitting fragment anteroinferiorly. The fibular fracture was classified into 4 types (Fig 5):

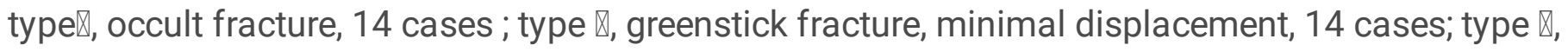

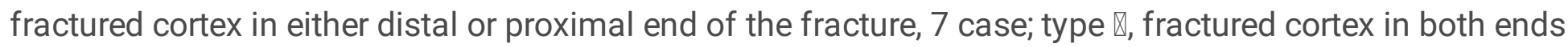
of the fracture, 5 cases. For those that involved the diaphysis, the fracture line was short spiral, without plantar flexion ( Fig 6).

\section{Discussion}

In our study, CT scans can well demonstrate the fracture orientation of the tibial metaphysis and differentiate the stages of these injuries for $\mathrm{S}-\mathrm{H} \otimes$ ankle fractures. We further deduce that the bending force plays an important role in resulting in the distal tibial metaphyseal fracture following SER injury when analyzing the fibular fracture in reconstructive CT views.

$\mathrm{CR}$ is the main method to diagnose the ankle fracture and has sufficient resolution in identifying fracture line and measuring the displacement of the fracture [8,12]. Still further, Dias and Tachdjian [5] classified the physeal fracture of the ankle in children into four mechanisms of injury after evaluating 58 ankle fractures. However, CR could not definitely demonstrate the precise mapping of the fracture plane orientation. In a retrospective review of pediatric triplane fractures, Eismann et al. [9] reported that the number or the direction of screws made by radiographs alone were changed in $41 \%$ of cases after reviewing CT. Therefore, knowledge of the axial fracture plane may be extremely useful to determine the optimal screw direction. Recently, a MRI study on distal tibial physeal fractures showed that the fracture line of the TH fragment on the axial view was within 10 degrees to the intermalleolar axis line which could be regarded as a guideline for insertion of the screws [18]. However, the intermalleolar axis line might be only a virtual concept. In our study, we defined the MFA between the fracture line of the distal tibia and the anterior tibial edge $0.5-1 \mathrm{~cm}$ proximal to the physis and found that the mean angle was 11 degrees which indicates that these two lines tends to be parallel in SER S-H $\otimes$ ankle fractures. When the trajectory of the screw is perpendicular to the anterior tibial edge, the fracture fragment could be compressed better. Our study showed that the mean length of the metaphyseal spike was $35 \mathrm{~mm}$ and this means that there is, if necessary, sufficient space to place the screws. So we believed that the anterior tibial edge is helpful clinically to plan screw insertion. However, the fracture plane varied in some cases, surgeons herein should analyze the axial CT images carefully to make a precise decision. 
Dias and Tachdjian pointed out that the first stage in the supination-plantar flexion and SER mechanism was radiologically quite similar [5]. Moreover, the distinction between SPF and SER injuries showed difficulties in radiological diagnosis when the fibular fracture was occult or non-displaced, especially the TH fragment being thinner and smaller [19] (Fig.4-A). The utility of CT can enhance the ability to detect fibular fracture [20]. In our study, there were fourteen cases with thin metaphyseal fragment and with absence of fibular fracture, so they were initially classified into SPF injury. After reviewing the CT images, the fragment was rotated posterolaterally and the fibula showed a green-stick fracture (Fig.4-B,C). These patients were thus classified into SER stage 2 injuries. This can facilitate reduction by reversing the direction of the initial force [5,21].

The ankle injuries both in adults and in children always occur in predictable stages and Dias and Tachdjian subdivided the pediatric ankle injuries into sequential grades [5,22]. The fibular fracture was an important radiographic marker for staging of the ankle injury. However, the mechanism of injury of the fibular fracture in pediatric injury has not been established yet. Most authors [5,20] believed that fibular fracture was suffered from more severe external rotational forces and characterized as spiral type starting medially and propagating posterosuperiorly in pediatric ankle injury, as stated by Lauge-Hansen in adults [22]. In our study, however, we only observed 4 patients who have shown this typical fracture and the location of the fracture line was situated in distal diaphysis.

In majority of the patients (40/44), a long oblique fracture of the distal fibular metaphysis was observed in present study. Moreover, the fibula showed significant plantar deformity with an anteroinferior fragment from the anterior fibular cortex. Karrholm et al. [23] interpreted that the injury in the final phase was primarily conditioned by bending force (plantar flexion). In our study, an interesting finding was that all these fibular fractures began at the level of the tibial physis and it was at the juxta-metaphysis rather than the weakest physis, that the fractures were located. The morphology of the fibular fracture was different from that in mature skeleton or adults (Fig. 7). As we all know, the fibula articulates with the tibiofibular joint and with the trochlea of the talus at the ankle joint. During the initial SER, the distal tibial epiphysis and the fibula move together as one entity via the intact and strong ligament system around the ankle following the separation of the tibial physis. As the rotatory movement continues, the distal tibial physis and the talus will make the fibular metaphysis compressed due to the peculiarity of extensive porosity [24] and thus plantar deforming force is believed to produce contributing to the instability of the ankle joint and the unique talar shape wider anteriorly than posteriorly. Then the force transmitted to the fibula and caused the fibular fracture. We separated the fibular fracture into 4 types based on the location and morphology of the fracture demonstrated on reconstructive CT views. Type $\nabla$ and $\nabla$ represented $70 \%$ of total fibular fractures that means most of the associated fibular fractures had minimal deformity. However, whether the energy of the bending force was gradually increasing remains unclear.

Furthermore, the upper limit of fibular fracture line was at the same or higher than the tip of TH fragment which probably confirmed that bending force was simultaneously exerted on the supinated foot at the time of injury. We also found that the fracture of MTC occurred more frequently in stage 1 than stage 2 
$(2.9 \%$ vs $45 \%, P=0.001)$. This may be explained that the displacement of the epiphyseal-metaphyseal fragment increased and MTC was broken following the fibular fracture.

Based on our observation, it may be helpful in clinical decision making. The reduction could be maintained without internal fixation due to the intact medial cortex being as a hinge that is akin to the green-stick forearm fracture, which significantly decreased the number of surgeries compared to those treated with internal fixation. In contrast, in cases with fractured medial cortex especially with associated type $\otimes$ fibular fracture, internal fixation, Kirschner pin or lag screw, would be added to maintain the reduction $[3,4,16]$.

The results of our study must be interpreted with the context of the study. First, the data were collected retrospectively, which introduced the possibility that CT scans might not be necessary in some cases. Second, CT does improve the understanding of S-H $\otimes$ distal tibial fractures and can better guide the surgeons to assess these fractures. This was more helpful in the situation where CT is not available. Despite this, CT was still not considered as a conventional stool for S-H $\otimes$ distal tibial fractures [25]. Third, the morphology of the fibular fracture in our study may be related to the activity patterns in our area.

In conclusion, this study demonstrated detailed information in CT scans for SER S-H $\otimes$ ankle fractures to guide the appropriate treatment plan. In addition to the external rotation force, there may be plantar flexion force based on the fibular morphology. Knowledge of the mechanism of the fibular fracture may make the reduction easier by reversing the original deforming force.

\section{Declarations}

Ethics approval and consent to participate in present study was approved by the Ethics Committee of Children's Hospital of Soochow University.

\section{Consent for publication}

Not applicable.

\section{Availability of data and material}

All data generated or analysed during this study are included in this manuscript.

\section{Competing interests}

The authors declare that they have no competing interests.

\section{Funding}

No funding was provided.

\section{Acknowledgements}


We thank all of the patients involved in the study.

\section{Authors' contributions}

QWY contributed to the study design, data analysis and interpretation, and manuscript draft.

FYZ, WL BBS and ZXG contributed to the data collection and analysis.

JFF and ZHZ contributed to the literature search.

YQL and XDW contributed to the analysis manuscript revision. All authors have read and approved the final manuscript.

ZYF contributed to the study design and is the corresponding author.

\section{Abbreviations}

S-H 叉: Salter-Harris type $\otimes$

SER: supination-external rotation

CR: conventional radiographs

CT: computed tomography

TH:Thurston-Holland fragment

MFA: metaphyseal fracture angle

MTC: medial tibial cortex

\section{References}

1. Landin LA, Danielsson LG. Children's ankle fractures. Classification and epidemiology. Acta Orthop Scand. 1983; 54(4):634-40.

2. Salter RB, Harris WR. Injuries involving the epiphyseal plate. J Bone Joint Surg. 1963; 45(3):587-622.

3. Seel EH, Noble S, Clarke NM, Uglow MG. Outcome of distal tibial physeal injuries. J Pediatr Orthop B. 2011; 20(4):242-8.

4. Leary JT, Handling M, Talerico M, Lin Yong, Bowe JA. Physeal fractures of the distal tibia: predictive factors of premature physeal closure and growth arrest. J Pediatr Orthop. 2009; 29(4):356-61.

5. Dias LS, Tachdjian MO. Physeal injuries of the ankle in children. Clin Orthop Relat Res.1978; 136:2303. 
6. Rohmiller MT, Gaynor TP, Pawelek J, Mubarak SJ. Salter-Harris I and II fractures of the distal tibia: does mechanism of injury relate to premature physeal closure? J Pediatr Orthop.2006;26(3):322-8.

7. Spiegel PG, Cooperman DR, Laros GS. Epiphyseal fractures of the distal ends of the tibia and fibula. A retrospective study of two hundred and thirty-seven cases in children. J Bone Joint Surg Am.1978; 60(8):1046-50.

8. Lemburg SP, Lilienthal E, Heyer CM. Growth plate fractures of the distal tibia: is CT imaging necessary? Arch Orthop Trauma Surg. 2010;130(11):1411-7.

9. Eismann EA, Stephan ZA, Mehlman CT, Denning J, Mehlman T, Parikh SN, Tamai J, Zbojniewicz A.

Pediatric triplane ankle fractures: impact of radiographs and computed tomography on fracture classification and treatment planning. J Bone Joint Surg Am, 2015,97(12):995-1002.

10. Pomeranz CB, Bartolotta RJ. Pediatric ankle injuries: utilizing the Dias-Tachdjian classification. Skeletal Radiol. 2020;49(4):521-30.

11. Jones S, Phillips N, Ali F, Fernandes JA, Flowers MJ, Smith TWD. Triplane fractures of the distal tibia requiring open reduction and internal fixation. Pre-operative planning using computed tomography. Injury. 2003;34(4):293-8.

12. Thawrani D, Kuester V, Gabos PG, Kruse RW, Littleton AG, Rogers KJ, Holmes L, Thacker MM. Reliability

and necessity of computerized tomography in distal tibial physeal injuries. J Pediatr Orthop.2011,31(7):745-50.

13. Horn BD, Crisci K, Krug M, Pizzutillo PD, MacEwen GD.Horn BD, et al. Radiologic evaluation of juvenile tillaux fractures of the distal tibia. J Pediatr Orthop. 2001;21(2):162-4.J Pediatr Orthop.

14. Cutler L Molloy A, Dhukuram V, Bass A. Do CT scans aid assessment of distal tibial physeal fractures? J Bone Joint Surg Br 2004;86:239-243.

15. Barmada A, Gaynor T, Mubarak SJ. Premature physeal closure following distal tibia physeal fractures: a new radiographic predictor. J Pediatr Orthop. 2003; 23(6):733-9.

16. Yuan QW, Zhen YF, Guo ZX, et al. Open reduction and internal fixation for displaced Salter-Harris type $\bigotimes$ fractures of the distal tibia: a retrospective study of sixty-five cases in children. . J Orthop Surg Res. 2021;16(1):224-30.

17. Hadad MJ, Sullivan BT, Sponseller PD. Surgically Relevant Patterns in Triplane Fractures: A Mapping Study. J Bone Joint Surg Am. 2018, 100(12):1039-46. 
18. Park J , Cha Y, Kang MS, Park SS. Fracture pattern and periosteal entrapment in adolescent displaced distal tibial physeal fractures: a magnetic resonance imaging study. J Orthop Trauma. 2019; 33(5):e196202.

19. Najaf-Zadeh A, Nectoux E, Dubos F, Happiette L, Demondion X, Gnansounou M, Herbaux B, Martinot A. Prevalence and clinical significance of occult fractures in children with radiograph-negative acute ankle injury. A meta-analysis. Acta Orthop. 2014;85(5):518-24.

20. Brown SD, Kasser JR, Zurakowski D, Jaramillo D. Analysis of 51 tibial triplane fractures using CT with multiplanar reconstruction. AJR Am J Roentgenol. 2004;183(5):1489-95.157.

21. Carothers $\mathrm{CO}$, Crenshaw $\mathrm{AH}$. Clinical significance of a classification of epiphyseal injuries at the ankle. Am J Surg, 1955;89(4):879-89.

22. Lauge-Hansen N.Fractures of the ankle. II. Combined experimental-surgical and experimentalroentgenologic investigations. Arch Surg. 1950;60(5):957-85.

23. Kärrholm J, Hansson LI, Laurin S. Supination-eversion injuries of the ankle in children: a retrospective study of radiographic classification and treatment. J Pediatr Orthop. 1982;2(2):147-59.

24. Ogden JA, McCarthy SM. Radiology of postnatal skeletal development. VIII. Distal tibia and fibula. Skeletal Radiol, 1983,10(4):209-20.

25. Frush DP, Donnelly LF, Rosen NS. Computed tomography and radiation risks: what pediatric health care providers should know. Pediatrics, 2003,112(4):951-7.

\section{Tables}

Table 1. Demographic data of $79 \mathrm{~S}-\mathrm{H}$ II distal tibial fractures. 


\begin{tabular}{ll} 
Variables & Results \\
\hline Gender & \\
\hline Female & 27 \\
\hline Male & 52 \\
\hline Injuried side & \\
\hline Left & 34 \\
\hline Right & 45 \\
\hline Age(y) & $12.2 \pm 1.4$ \\
\hline MFA $\left(^{\circ}\right)$ & $11.2 \pm 9.1$ \\
\hline Size of TH fragment & $35.3 \pm 12.1$ \\
\hline Stage of injury & \\
\hline stage 1 & 35 \\
\hline stage 2 & 44
\end{tabular}

MFA, metaphyseal fracture angle; TH, Thurston-Hollandfragment.

Table 2. Comparison of stage 1 and stage 2 among several parameters.

Variables $\left.\quad \mathrm{MFA}^{\circ}\right) \quad$ Size of the TH fragment $\rrbracket \mathrm{mm} \rrbracket \quad$ MTC

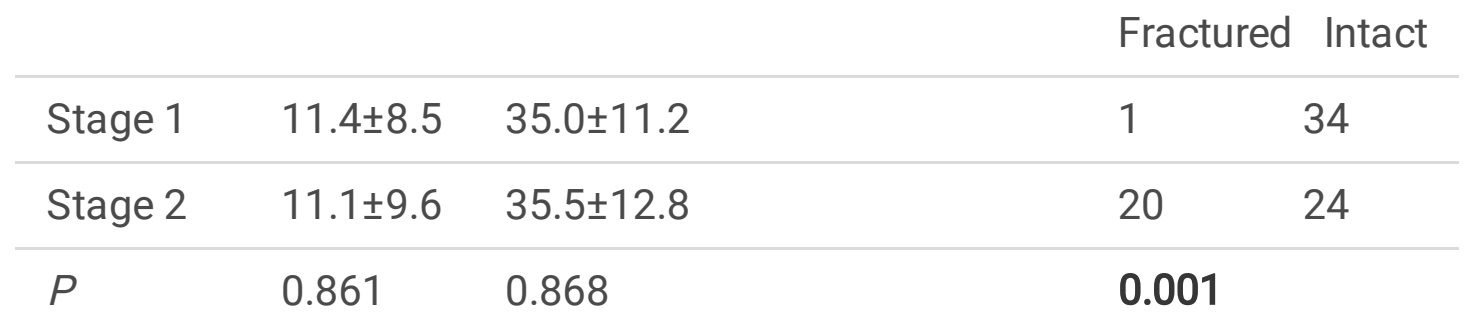

MFA, metaphyseal fracture angle; $\mathrm{TH}$, Thurston-Holland. MTC=medial tibial cortex.

\section{Figures}




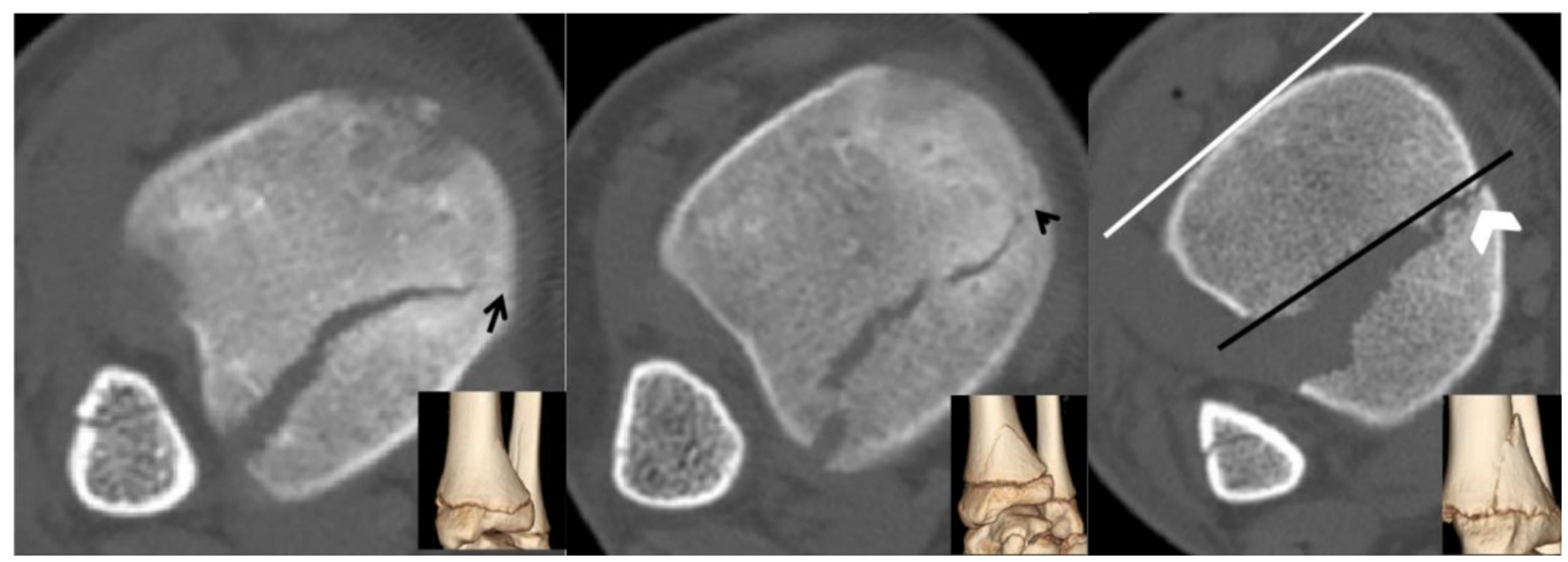

\section{Figure 1}

Example of conditions of MTC in axial CT image and the metaphyseal angle. A, 10.5-year-old girl. The axial CT image shows the intact MTC (arrow). B, 10-year-old boy. The axial CT image shows MTC was not fully penetrated (arrowhead). C, 12-year-old boy. The axial CT image shows MTC was completely fractured (chevron). The white solid line and the black solid line represent the anterior edge and the metaphyseal fracture plane of the distal tibia, respectively. These findings were confirmed in reconstructive CT images (at the bottom of the each corresponding axial image). MTC, medial tibial cortex.

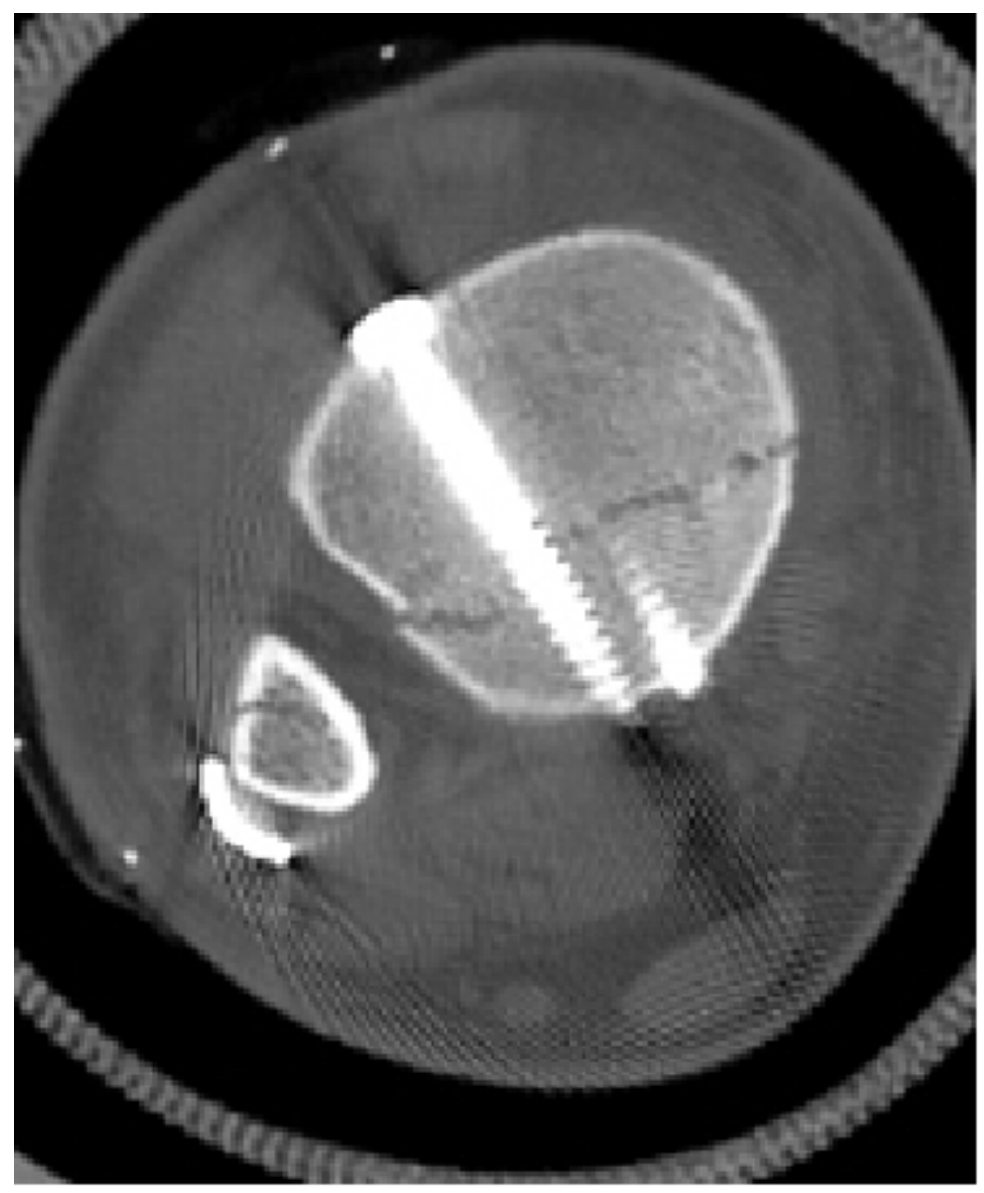


Figure 2

the same patient with Fig 1-C. The axial CT image postoperatively displays the exact reduction of the fracture and direction of the screws.

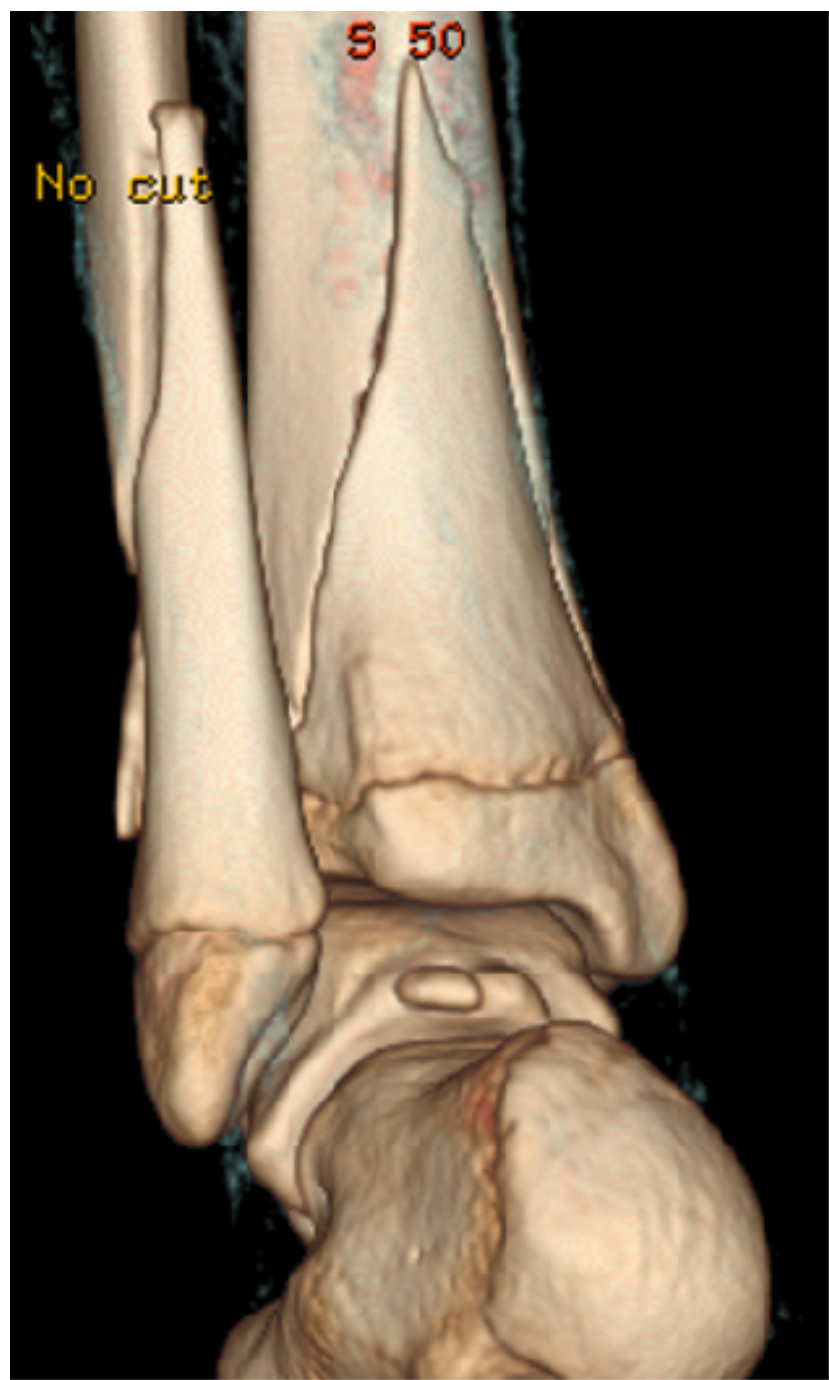

Figure 3

12.4-year-old girl. A reconstructive CT image shows that both upper ends of fibular fracture and the TH fragment are at the same level. TH,Thurston-Holland. 


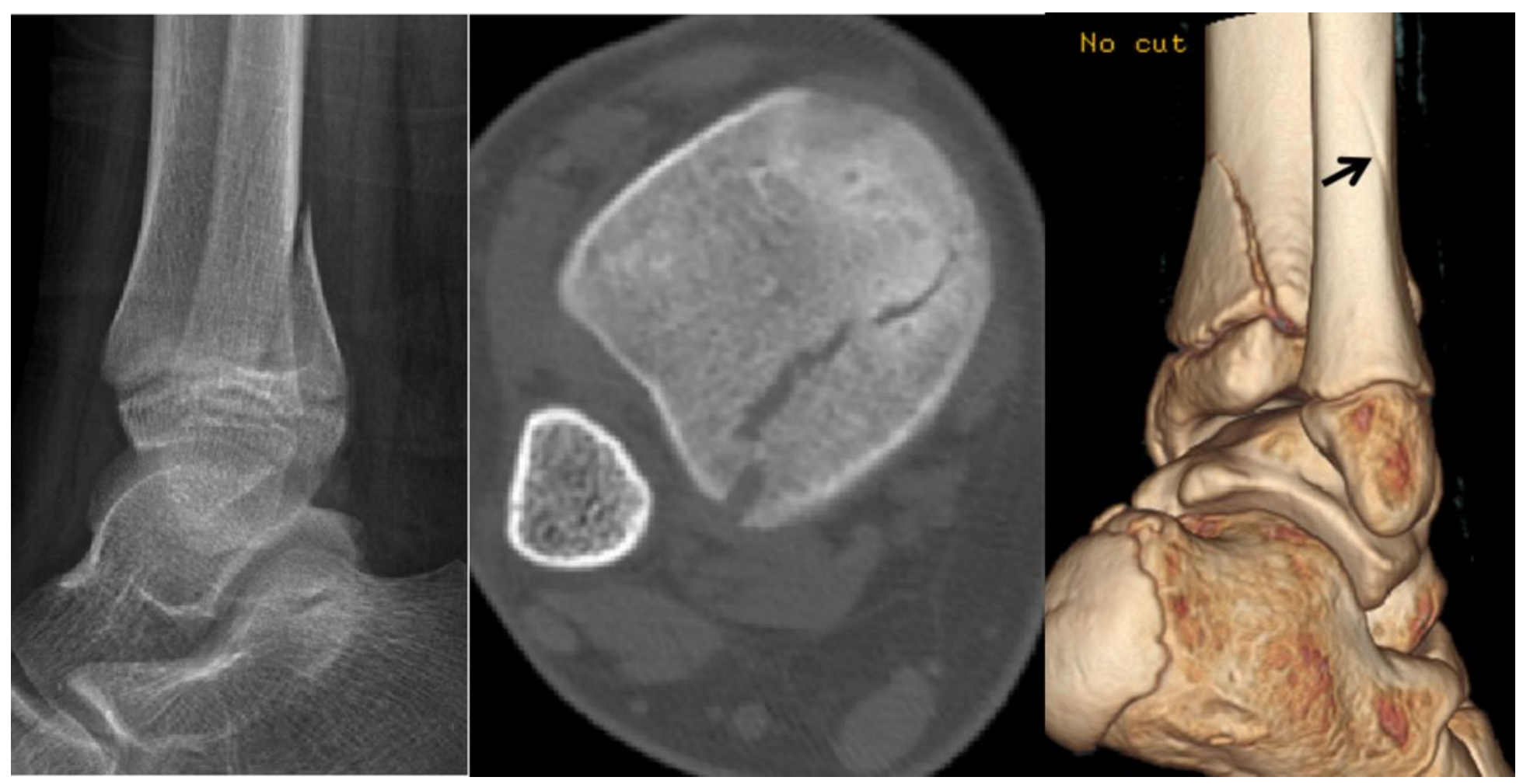

Figure 4

10-year-old boy. The differentiation between the mechanism of SER andsupination-plantar flexion injury. A, Lateral radiograph shows a thin posterior methaphyseal spike and no fibular fracture. The injury may be classified into supination-plantar flexion. B and C, The axial CT image (B) shows the posterolateral rotation of the metaphyseal fragment and and the reconstructive $\mathrm{CT}$ image (C) demonstrates the fracture line in distal fibula (black arrow). The injury mechanism is classified into SER. SER,supination-external rotation

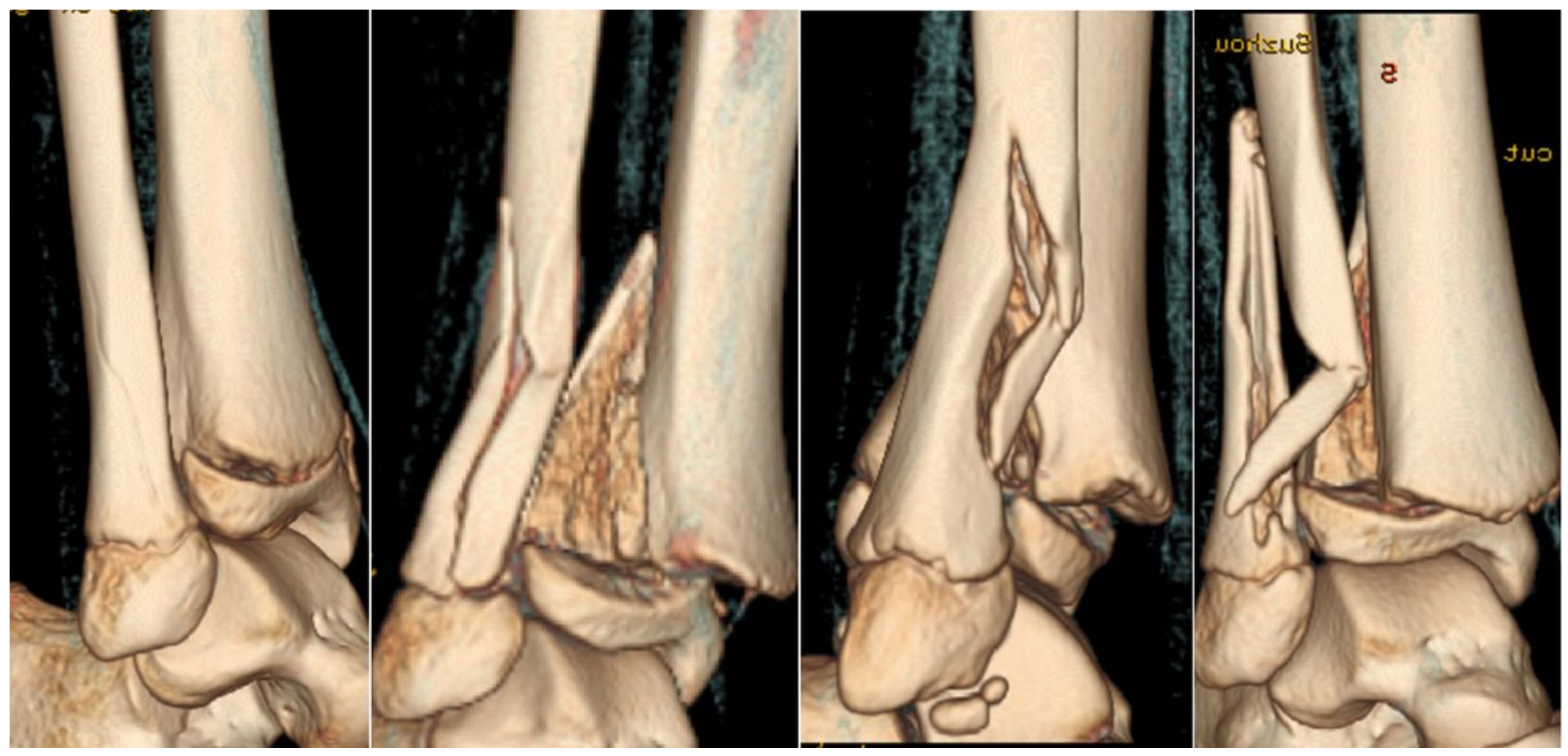




\section{Figure 5}

The four patterns of the fibular fracture located at the metaphysis. A, Type邓- occult fracture (11-year-old

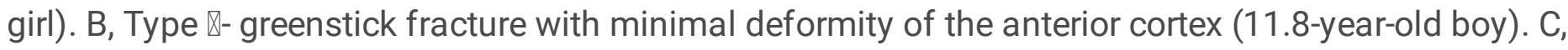
Type $\varangle$ - fractured cortex in either distal or proximal end of the fracture with moderate deformity of the anterior cortex (12-year-old boy). D, Type 》-fractured cortex in both ends of the fracture with severe deformity and an anteroinferior fragment (12.4-year-old girl).

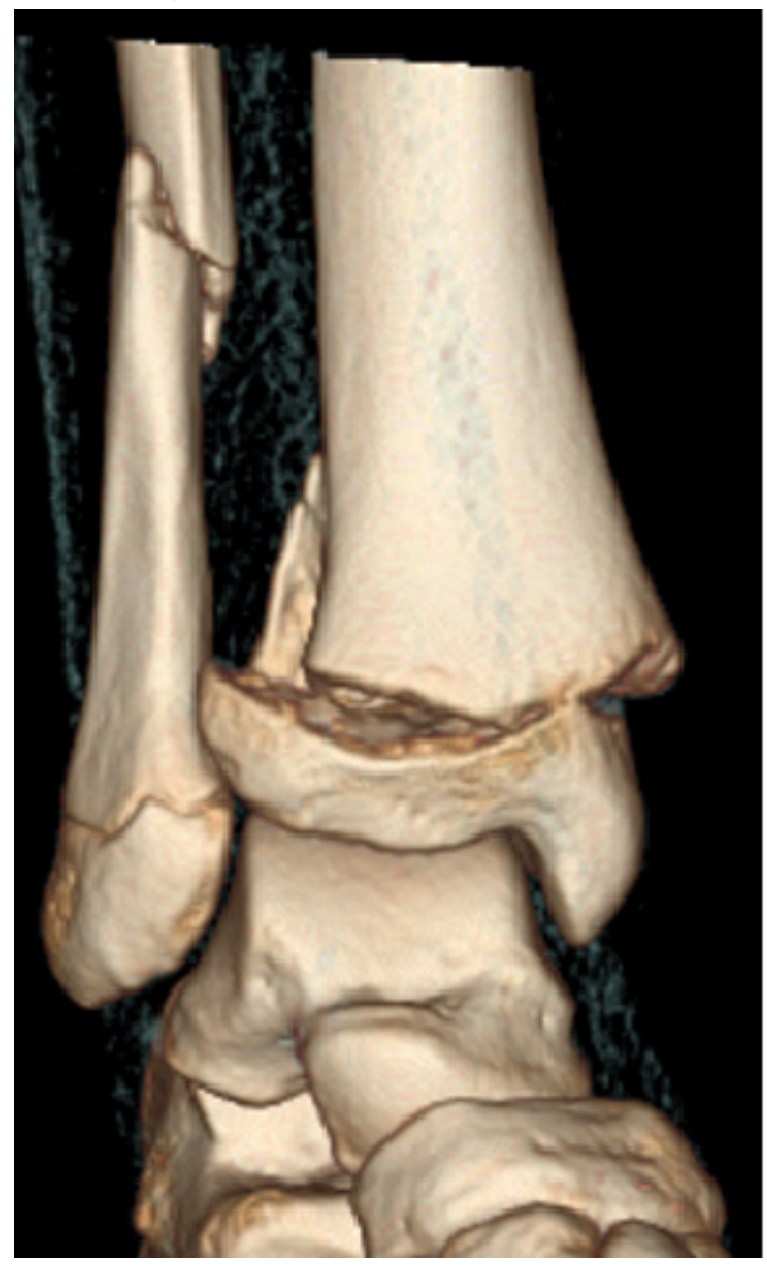

Figure 6

14.5-year-old boy. A reconstructive CT image shows a spiral fracture located at the distal diaphysis without deformity of the anterior cortex. 


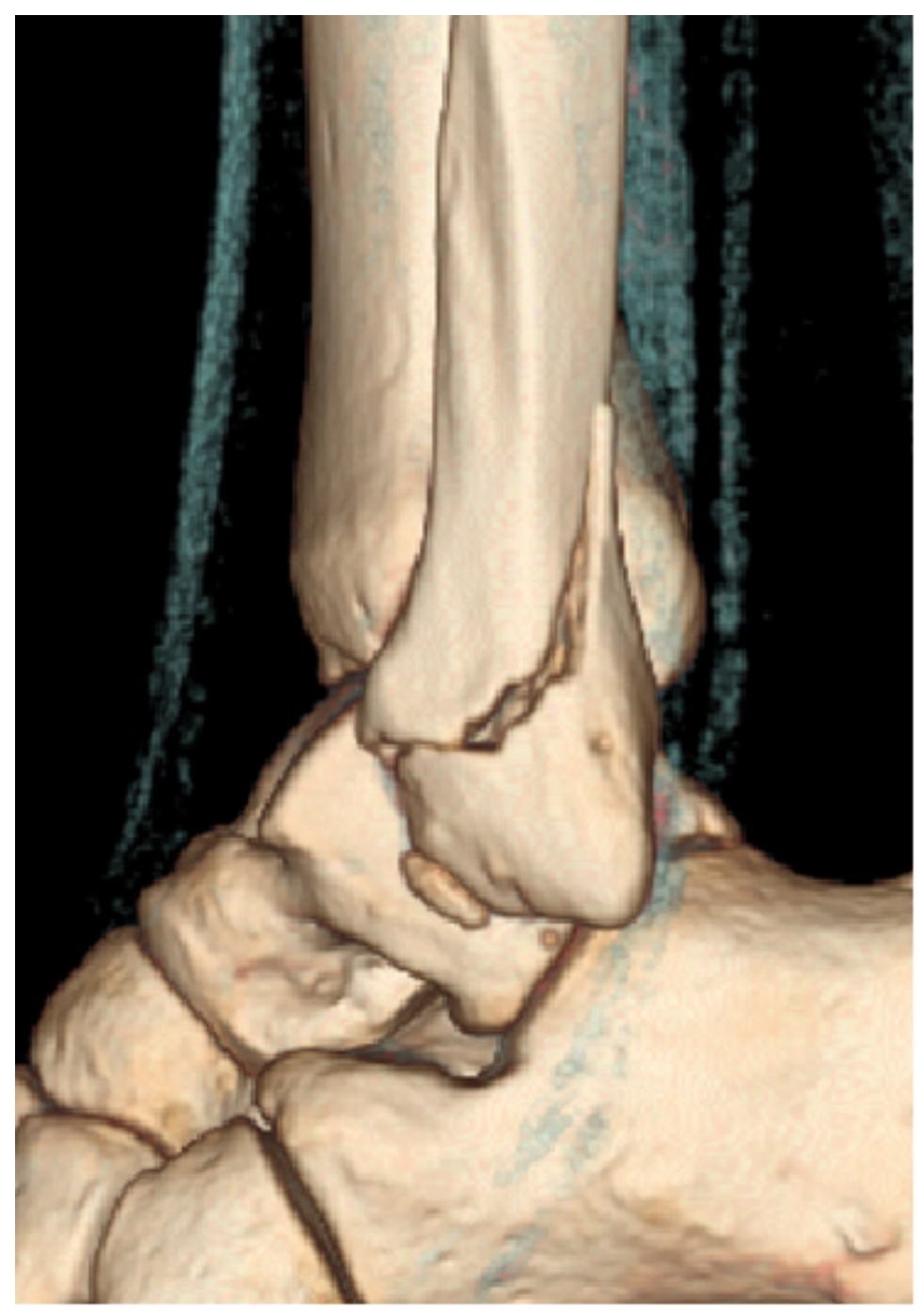

\section{Figure 7}

15-year-old boy, with closed distal tibial and fibular physis. The reconstructive CT image shows that the fibular fracture line propagated posterosuperiorly without plantar flexion. 